Cahiers de la recherche sur les droits

Cahiers

${ }^{\text {sur les }}$ Droits fondamentaux

$2 \mid 2003$

Les titulaires particuliers des droits fondamentaux

\title{
Les droits des personnes incarcérées : entre punition et réhabilitation
}

Jean-Manuel Larralde

\section{(2) OpenEdition}

1 Journals

Édition électronique

URL : https://journals.openedition.org/crdf/7712

DOI : $10.4000 /$ crdf. 7712

ISSN : 2264-1246

Éditeur

Presses universitaires de Caen

Édition imprimée

Date de publication : 1 janvier 2003

Pagination : 63-76

ISBN : 2-84133-205-5

ISSN : $1634-8842$

Référence électronique

Jean-Manuel Larralde, «Les droits des personnes incarcérées : entre punition et réhabilitation », Cahiers de la recherche sur les droits fondamentaux [En ligne], 2 | 2003, mis en ligne le 18 décembre 2020, consulté le 14 novembre 2022. URL : http://journals.openedition.org/crdf/7712 ; DOI : https:// doi.org/10.4000/crdf.7712 


\title{
Les droits des personnes incarcérées: entre punition et réhabilitation
}

\author{
Jean-Manuel LARRALDE \\ Maître de conférences en droit public \\ Université de Caen Basse-Normandie
}
I. L'émergence d'un détenu sujet de droit
A. L'évolution des conceptions carcérales et ses répercussions sur les droits des personnes incarcérées
B. La progression de la légalité en prison

II. Droits des détenus et réalités pénitentiaires

A. Le maintien des spécificités du droit pénitentiaire

B. L'exercice des droits du détenu face à la réalité carcérale

Jamais, depuis l'instauration de cette sanction pénale au cour du dispositif répressif français ${ }^{1}$, la prison n'aura été l'objet de tant de regards extérieurs qu'au cours de ces dernières années. Le rapport du premier Président de la Cour de cassation sur les exigences de contrôle des établissements pénitentiaires ${ }^{2}$, le livre éminemment médiatisé de l'ancien médecin-chef de la prison de la Santé ${ }^{3}$, ou les deux rapports des commissions d'enquête parlementaire $^{4}$, ont tous participé à un ample mouvement de réflexion sur la prison et ses missions. Au-delà de la dénonciation des multiples dysfonctionnements de l'institution pénitentiaire, cette médiatisation, presque inédite sous la $\mathrm{V}^{\mathrm{e}}$ République $^{5}$, a également permis de s'interroger sur les missions et objectifs de la prison et sur la place du détenu dans le dispositif carcéral. La prison constitue une institution complexe, au confluent de plusieurs théories, souvent ambivalentes, et qui entraînent une multiplication des missions que l'Administration pénitentiaire doit faire coexister. Comme le Conseil constitutionnel a pu le rappeler, «l'exécution des peines privatives de liberté en matière correctionnelle et criminelle a été conçue, non seulement pour protéger la société et assurer la punition du condamné, mais aussi pour favoriser l'amendement de celui-ci et préparer son

1. Loi du 16 septembre 1791.

2. G. Canivet, L'Amélioration du contrôle extérieur des établissements pénitentiaires, Rapport remis à Madame le garde des Sceaux, ministre de la Justice, 6 juillet 2000 .

3. V. Vasseur, Médecin-chef à la prison de la Santé, Paris, Le Cherche Midi, 2000.

4. Rapport de M. J. Floch sur la situation dans les prisons françaises, Assemblée nationale, rapport 2251, 28 juin 2000,2 tomes, et Rapport de M. G.-P. Cabanel, Prisons : une humiliation pour la République, rapport de la Commission d'enquête du Sénat, rapport 449, t. I, 28 juin 2000.

5. Depuis 1958, la seule interrogation de grande ampleur sur la prison a été initiée par le Groupe d'information sur les prisons, créé en 1971 par Jean-Marie Domenach, Pierre Vidal-Naquet et Michel Foucault. 
éventuelle réinsertion ${ }^{6}$. Ces différentes perceptions de la prison ${ }^{7}$ influencent directement la situation de celui qui doit être au cœur du dispositif répressif: le détenu. On va ainsi découvrir progressivement que celui-ci ne doit pas seulement être un objet de punition, mais qu'il doit aussi être réhabilité, " corrigé », en vue de son retour, à terme, dans la société. L'article $1^{\text {er }}$ de la Charte de la réforme pénitentiaire de 1945 prévoit que «la peine privative de liberté a pour objet essentiel l'amendement et le reclassement social du condamné». De même, l'article 728 du Code de procédure pénale (CPP) édicte : «Dans les prisons établies pour peines, le régime sera institué en vue de favoriser l'amendement des condamnés et de préparer leur reclassement social. » Amender et reclasser le condamné, cela implique qu'un individu en prison ne perd en aucun cas son statut de sujet de droit. Or, une telle analyse théorique n'apparaît que récemment ${ }^{8}$ et il faudra attendre (à la suite de divers conflits et de crises) la décennie 1970 pour que les détenus se voient reconnaître progressivement des droits, dont la liste ne cessera de s'étendre jusqu'à aujourd'hui ${ }^{9}$. Les débats relatifs à la reconnaissance de droits au profit des personnes incarcérées pourraient sembler épuisés, si l’on estime que la peine privative de liberté ne constitue justement qu'une privation de liberté que l'Administration pénitentiaire devrait mettre à profit pour amender le délinquant. Comme avait pu le dire en d'autres termes l'ancien président de la République Valéry Giscard d'Estaing dans une formule devenue célèbre : «la peine, c'est la détention, et donc, ce n'est pas plus que la détention ${ }^{10}$. Or les choses sont plus complexes, car le caractère afflictif de la peine, les exigences de sécurité, la composition sociologique de la population carcérale, la situation matérielle des établissements pénitentiaires constituent autant de variables qui doivent être prises en considération, au même titre que la progression du droit positif stricto sensu.

L'époque contemporaine est bien celle de l'avènement d'un détenu sujet de droit, ce qui s'est caractérisé par la création progressive d'un véritable statut, fixant des droits et des obligations, auxquels il faut attacher la maîtrise de voies de recours permettant de les faire respecter (I). Ce constat fortement optimiste doit toutefois être immédiatement tempéré. La prison reste un milieu clos, présentant des particularités marquées, qui influencent à la fois la structure et la conception des droits reconnus aux personnes incarcérées, mais également leur exercice concret dans les établissements (II).

\section{L'émergence d'un détenu sujet de droit}

Définir et délimiter les droits des personnes incarcérées, c'est aussitôt renvoyer aux conceptions que l'on se fait de la prison, tant les différentes missions que l'on peut lui confier peuvent influencer les droits reconnus aux prisonniers (A). Ces droits ne peuvent néanmoins en aucun cas rester de simples références textuelles et le détenu, sujet de droit, n'existe que par un renforcement de la légalité en prison (B).

\section{A. L'évolution des conceptions carcérales et ses répercussions sur les droits des personnes incarcérées}

La reconnaissance de droits au profit de personnes incarcérées constitue une question indissociable des buts assignés à la peine privative de liberté. Or, l'enfermement carcéral se situe au confluent de plusieurs logiques, qui participent de raisonnements différents. L'époque moderne, qui naît avec le XIX ${ }^{\mathrm{e}}$ siècle, est porteuse d'idées multiples en ce qui concerne les fondements de la sanction carcérale. Elle est la "phase des contradictions " ${ }^{11}$, essayant de concilier des principes a priori aussi divergents que ceux de la réhabilitation par un traitement approprié, de la prévention par intimidation et de la protection de la société à l'égard de ses membres jugés les plus dangereux ${ }^{12}$. Enfermer un être humain pour le punir, c'est donc tout à la fois considérer qu'on peut le corriger, le normaliser ou le réinsérer... C'est également dissuader les éventuels criminels et protéger la société. Pour J. Foyer, ancien garde des Sceaux français, «s'il est hors de doute qu'il est indispensable de se préoccuper de l'amendement et du reclassement social du condamné, il ne faut pas perdre de vue le caractère intimidant qui souhaite s'exercer à la fois sur le condamné lui-même et sur les autres ${ }^{13}$. On se trouve donc bien face à une ambiguïté qui va largement limiter la portée et l'efficacité du traitement pénitentiaire. La prison doit tout faire pour rendre dans les meilleures conditions possibles le détenu

6. Décision n ${ }^{\circ}$ 93-334 DC du 24 janvier 1994, Loi instituant une peine incompressible et relative au nouveau code pénal et à certaines dispositions de procédure pénale. Voir T. S. Renoux, Revue française de droit constitutionnel, 1994, p. 353.

7. Sur ce point, voir notamment P. Combessie, Sociologie de la prison, Paris, La Découverte (Repères), 2001, p. 14 sq.

8. À tel point qu'un membre éminent de la doctrine belge, J. Dupreel, peut écrire en 1960 un article intitulé: «Une notion nouvelle, les droits des détenus ", Études et Perspectives pénitentiaires, 1960, p. 87 sq.

9. Voir J. Favard, Des prisons, Paris, Gallimard (Au vif du sujet), 1987, p. 20 sq.

10. Déclaration du 25 juillet 1974 .

11. D. Szabo, Criminologie, Montréal, Presses de l'Université de Montréal, 1965, p. 449.

12. Cette ambiguïté a d'ailleurs été également soulevée par E. Durkheim : «... Pour se faire une idée exacte de la peine, il faut réconcilier les deux théories contraires qui en ont été données; celle qui y voit une expiation et celle qui en fait une arme de défense sociale. Il est certain, en effet, qu'elle a pour fonction de protéger la société, mais c'est parce qu'elle est expiatoire; et d'autre part, si elle doit être expiatoire, ce n'est pas que, par la suite de je ne sais quelle vertu mystique, la douleur rachète la faute, mais c'est qu'elle ne peut produire son effet socialement utile qu'à cette condition" (De la division du travail social, $2^{\mathrm{e}}$ éd., Paris, PUF (Quadrige), 1991, p. 77).

13. J. Foyer, «La doctrine de la $\mathrm{V}^{\mathrm{e}}$ République en matière de répression », Revue pénitentiaire et de droit pénal, nº 2, 1963, p. 286. 
à la société, et non le désocialiser en ne lui infligeant qu'une peine dure et afflictive.

Le détenu moderne n'est plus seulement la personne qui doit payer une dette, en raison d'une faute commise. La société qui punit le délinquant se voit désormais imposer un certain nombre de devoirs, ce qui constitue une nouveauté considérable. Loin d'être jeté dans un cul de basse fosse à l'écart du monde, le prisonnier doit, au contraire, être aidé progressivement à réintégrer une société dont il n'a été que momentanément écarté. Pour ce faire, le régime pénitentiaire doit se rapprocher, autant que faire se peut, de la vie libre. L'article D 241 du CPP prévoit d'ailleurs que la situation de la personne détenue est précisément régie, dans sa vie physique comme dans sa vie spirituelle, sans que jamais elle doive souffrir d'une quelconque discrimination, à quelque titre que ce soit. Les seules restrictions admises à l'exercice des droits doivent être motivées par des exigences liées à la discipline et à la sécurité de l'établissement pénitentiaire.

L'approche qui prévaut est que désormais, le délinquant doit posséder tous les droits de l'homme libre, hormis ceux dont il doit être privé " pour la protection de la société ${ }^{14}$. Selon la théorie contemporaine, le détenu ne devient pas une personne en dehors du droit pendant son incarcération. Bien plus, le condamné incarcéré n'est pas déchu des droits autres que ceux attachés à la privation de liberté, à l'exception de ceux expressément retirés par le jugement de condamnation ${ }^{15}$. Cette conception a été notamment exprimée par la section britannique de la Commission internationale de juristes en 1983: «Un détenu perd son droit à la liberté et ses autres droits sont restreints dans la mesure où ils sont incompatibles avec cette privation de liberté et avec l'obligation de vivre dans une prison soumise à une discipline propre. Ceci est indéniable. Il est non moins évident qu'il conserve tous ses autres droits, affectés seulement par cette restriction nécessaire et qu'il acquiert même de nouveaux droits à l'encontre de l'État qui l'emprisonne ${ }^{16}$. Comme l'indique plus récemment $\mathrm{M}$. Guy Canivet, «pour résoudre le paradoxe qui consiste à réinsérer une personne en la retirant de la société, il n'y a d'autre solution que de rapprocher autant que possible la vie en prison des conditions de vie à l'extérieur, la société carcérale de la société civile ${ }^{17}$.

Les normes juridiques formant le «droit pénitentiaire» sont évidemment porteuses de ces différentes options et l'on retrouve dans plusieurs textes mention des buts et fonctions de la prison. L'un des plus ambitieux à cet égard est probablement celui de la loi canadienne sur le système correctionnel et la mise en liberté sous condition (1992 ch. 20) qui, dans son article 3, définit ainsi la mission du système correctionnel (et non "pénitentiaire »!) : «Le système correctionnel vise à contribuer au maintien d'une société juste, vivant en paix et en sécurité, d'une part, en assurant l'exécution des peines par des mesures de garde et de surveillance sécuritaires et humaines, et d'autre part, en aidant au moyen de programmes appropriés, dans les pénitenciers ou dans la collectivité, à la réadaptation des délinquants et à leur réinsertion sociale à titre de citoyens respectueux des lois ${ }^{18}$.

Le système français actuel n'a pas véritablement tranché entre une prison strictement punitive et éliminatrice ou bien correctrice et réhabilitatrice. Le sens de la peine n'a d'ailleurs jamais constitué un véritable enjeu du débat politique national. L'administration pénitentiaire se trouve dès lors obligée de gérer un système sans pouvoir s'appuyer sur une véritable définition de ses missions. La loi du 22 juin 1987 relative au service public pénitentiaire a ainsi partiellement défini la double mission de ce service public, d'exécution des décisions de justice et de « réinsertion sociale des personnes qui lui sont confiées par l'autorité judiciaire », en précisant qu'il est organisé "de manière à assurer l'individualisation des peines". Selon l'article D 189 du CPP, à l'égard de toutes les personnes « confiées par l'autorité judiciaire, à quelque titre que ce soit, le service public pénitentiaire assure le respect et la dignité inhérente à la personne humaine et prend toutes les mesures destinées à faciliter leur réinsertion sociale». Cette double mission, formulée en des termes on ne peut plus généraux, a néanmoins permis au détenu actuel une reconnaissance progressive de droits, n'en faisant plus un individu totalement retiré de la société.

Le traitement pénitentiaire contemporain implique non seulement le respect de la dignité de l'homme puni, la protection de sa santé physique et mentale, de son développement intellectuel et sportif, le maintien des liens familiaux et sociaux, mais également la promotion de la resocialisation au rang de véritable droit du détenu ${ }^{19}$. La vie en prison doit être organisée de telle manière que la privation de liberté ne soit pas aggravée par les conditions anormales dans lesquelles elle est subie. Ce droit aujourd'hui primordial conditionne lui-même l'existence de certains droits qui s'avèrent indispensables pour rendre le traitement effectif.

C'est à partir des années 1980 que le droit français de la prison a connu une forte rénovation, par des textes qui ont octroyé des droits multiples aux personnes détenues. C'est en particulier le cas du décret du 26 janvier 1983, complété par un décret du 6 août 1985 (qui modifie une centaine d'articles du Code de procédure pénale), qui a modernisé et humanisé les prisons françaises dans un processus de «socialisation carcérale ${ }^{20}$. Le droit français,

14. P. Cornil, «Les problèmes de droit pénal appliqué et les nouvelles tendances en la matière », Revue de droit pénal et de criminologie, 1951, p. 494 sq.

15. Voir, notamment, M. Ancel et P. Chemithe, Les Systèmes pénitentiaires en Europe occidentale, Paris, La documentation française (Notes et études documentaires), 1981, p. 29 sq.

16. Cité par S. Shaw, in Human rights in the U.K., P. Sieghart (éd.), Londres, Pinter Pub., 1988, p. 40.

17. Cité par G. Canivet, L'Amélioration du contrôle..., p. 2.

18. Source: http://lois.justice.gc.ca/fr/C-44.6/.

19. Voir J.-M. Varaut, La Prison, pour quoi faire?, Paris, La Table ronde, 1972, p. 196 sq.

20. J.-C. Froment, «Vers une prison de droit? », Revue de science criminelle et de droit pénal comparé, 1997, p. 539. 
influencé par les instruments internationaux ${ }^{21}$, présente désormais les principaux éléments d'un statut de la personne incarcérée, ce qui passe par plusieurs axes de protection $^{22}$.

Désormais, les locaux de détention, et en particulier ceux qui sont destinés au logement, doivent « répondre aux exigences de l'hygiène, compte tenu du climat, notamment en ce qui concerne le cubage d'air, l'éclairage, le chauffage et l'aération » (art. D 350 CPP), chaque détenu disposant «d'un lit individuel et d'une literie appropriée » (art. D 356 CPP). Les installations sanitaires doivent être "propres et décentes" (art. D $351 \mathrm{CPP}$ ) et chaque détenu a droit à trois douches au moins par semaine (art. D 358 CPP). L'alimentation des détenus doit être «variée, bien préparée et présentée, répondant tant en ce qui concerne la qualité que la quantité aux règles de la diététique et de l'hygiène...» (art. D 354 CPP). Quant aux vêtements et sous-vêtements des détenus ils doivent être " propres et maintenus en bon état » (art. D 355 CPP). C'est le comportement personnel du détenu qui a également évolué avec la reconnaissance progressive de droits touchant à l'habillement (art. D 348 $\mathrm{CPP}$ ), à la tenue personnelle (droit de conserver les cheveux longs, la barbe ou la moustache: art. D $358 \mathrm{CPP}$ ), à l'accès aux médias (journaux sans censure en 1970, radio en 1974 et télévision en 1985), à la vie quotidienne (suppression de l'extinction des lumières avec la circulaire du 27 décembre 1988)

La protection de la personne du détenu passe évidemment par le dispositif de soins, profondément rénové par les articles 2 à 7 de la loi du 18 janvier 1994 relative à la santé publique et à la protection sociale (complétée par le décret du 27 octobre 1994 et la circulaire interministérielle du 8 décembre 1994). Cette réforme a constitué un progrès énorme et a considérablement amélioré les standards de qualité des soins apportés aux détenus. La loi de 1994 a permis de «faire rentrer l'hôpital dans les prisons ${ }^{23}$. Le législateur a eu pour objectif d'intégrer la population pénale dans le système de santé selon deux axes principaux : le premier qui est d'accorder au détenu une couverture sociale, le second de lui permettre d'accéder à des soins comparables à ceux dispensés en milieu libre, au travers du service public hospitalier. De même, le droit à une affiliation à la sécurité sociale a été reconnu, pour les détenus et leurs ayants droit, bénéfice maintenu pendant un an à compter de leur libération (art. L 412-8-5 du Code de la Sécurité sociale), comme plus généralement celui du droit à la protection sociale (art. D 366 sq. CPP). Dans la même optique, le bénéfice des prestations familiales et de l'assurance vieillesse leur a été accordé (art. L 521-1 et L 381-31 du Code de la Sécurité sociale). Ces droits ont correspondu à la volonté législative d'étendre la protection sociale à tous les citoyens en considérant comme tels les personnes détenues, mais aussi de favoriser la réinsertion sociale des condamnés. Plus récemment, la loi du 4 mars 2002 a également prévu la possibilité d'une suspension de peine qui peut "être ordonnée, quelle que soit la nature de la peine ou la durée de la peine restant à subir, et pour une durée qui n'a pas à être déterminée, pour les condamnés dont il est établi qu'ils sont atteints d'une pathologie engageant le pronostic vital ou que leur état de santé est durablement incompatible avec le maintien en détention, hors les cas d'hospitalisation des personnes détenues en établissement de santé pour troubles mentaux» (nouvel art.720-1-1 $\mathrm{CPP})^{24}$. Cette disposition devra probablement jouer un rôle important dans l'évolution des peines, sous l'influence de la Cour européenne des droits de l'homme qui a estimé que la France avait violé l'article 3 de la Convention européenne des droits de l'homme pour avoir tardé à libérer un détenu atteint d'une leucémie et l'avoir soumis au port des menottes et des entraves de façon « disproportionnée $»^{25}$.

Les détenus sont également définis comme des individus qui ne sont que temporairement retirés de la société dans laquelle ils vivent, qu'ils soient prévenus ou condamnés. Le maintien de liens avec l'extérieur est donc organisé. Ils peuvent ainsi correspondre par écrit librement avec toute personne de leur choix, sans limitation (art. D 65, D 413 et D 417 CPP), les courriers qu'ils échangent avec leurs avocats étant couverts par le secret (art. D 69 et D 419 CPP). S'agissant de l'utilisation du téléphone, les condamnés incarcérés dans les établissements pour peines peuvent être autorisés à téléphoner, «dans des circonstances familiales ou personnelles importantes ", tandis que dans les centres de détention, ils sont «autorisés à téléphoner une fois par mois... aux membres de leur famille ou aux titulaires de permis de visites» (art. D 417, al. 2 et $3 \mathrm{CPP})^{26}$. Les détenus ont aussi le droit de recevoir des visites sur autorisation du

21. Sur l'influence du droit européen sur le droit pénitentiaire français, voir notre article, «Le soft law européen comme promoteur des droits des personnes incarcérées », in Questions sur le droit européen, C. Grewe (dir.), Caen, Presses universitaires de Caen, 1996, p. 189-208.

22. Pour une présentation plus détaillée des droits des personnes incarcérées, voir G. Canivet, L'Amélioration du contrôle..., p. 12 sq.; O. Clingman, L. Gratiot, J.-C. Hanoteau, Le Droit en prison, Paris, Dalloz, 2001; Le Nouveau Guide du prisonnier, Paris, Observatoire international des prisons Éditions de l'Atelier, 2000.

23. Voir, notamment, Prisons: une humiliation pour la République.

24. Cette disposition a connu une médiatisation considérable lors de la libération de M. Papon en septembre 2002. Voir notamment, note M. HerzogEvans, «La suspension de peine médicale de Maurice Papon», Dalloz, 2002, p. 2893 sq.; «Le cas des détenus octogénaires », Le Monde, 29 septembre 2002 ; C. Simonnot, «Les détenus s'emparent de Papon», Libération, 15 octobre 2002.

25. CEDH, 14 octobre 2002, Mouisel c. France. Voir J.-P. Céré, «Le maintien en détention de malades graves constitue un traitement inhumain et dégradant», Revue trimestrielle des droits de l'homme, ${ }^{\circ}$ 55, 2003, p. 1007 sq. ; H. Tigroudja, Les Petites Affiches, 19 juin 2003 , p. 18 sq.

26. La loi du 9 septembre 2002 (dite loi Perben) dans son article 47 a prévu le contrôle de l’introduction des téléphones portables en prison, en installant des systèmes de brouillage radioélectrique, afin de les rendre inopérants dans l'enceinte des établissements pénitentiaires, ceci tant en ce qui concerne l'émission que la réception. Voir J.-P. Céré, «La loi n ${ }^{\circ}$ 2002-1138 du 9 septembre 2002 sur l’amélioration du fonctionnement et de la sécurité des établissements pénitentiaires », Dalloz, 2002, «chronique», p. 3225. 
magistrat instructeur s'ils sont prévenus (art. D 64 CPP), du chef d'établissement s'ils sont condamnés (art. D 403 $\mathrm{CPP}$ ). L'exercice matériel des visites est uniformément fixé pour les prévenus comme pour les condamnés (art. D 403 sq. CPP) et elles s'opèrent normalement par le biais de parloir sans dispositif de séparation, sauf incident particulier réel ou redouté (art. D 405 CPP). Membres de la société malgré la condamnation, les détenus restent enfin des citoyens qui, dès lors qu'ils ne sont pas privés du droit de vote par leur condamnation, peuvent participer à l'exercice du suffrage : depuis 1975, le législateur accorde aux détenus la possibilité de voter par procuration.

Le monde pénitentiaire n'est pas resté à l'écart du développement d'un droit du travail. Dès 1975, les détenus ont obtenu que leur salaire soit calculé selon le travail accompli et non plus selon leur comportement ou leur statut pénal. Le travail constitue désormais un élément de la préparation à la réinsertion sociale et la loi du 22 juin 1987 a supprimé l'obligation au travail, en précisant qu' " au sein des établissements pénitentiaires, toutes dispositions sont prises pour assurer une activité professionnelle aux personnes incarcérées qui le souhaitent ». Selon l'article D 101 al. 2 du CPP : «Dans la mesure du possible, le travail de chaque détenu est choisi en fonction non seulement de ses capacités physiques et intellectuelles, mais encore de l'influence que ce travail peut exercer sur les perspectives de sa réinsertion. » L'organisation, les méthodes et les rémunérations du travail doivent «se rapprocher autant que possible de celles des activités professionnelles extérieures » (art. D 102 CPP), le droit à la réparation des accidents du travail et des maladies professionnelles devant être reconnu aux détenus (art. D 110 CPP).

Enfin, plusieurs droits visent à assurer l'accès aux activités culturelles et socioculturelles (art. D 443 à D 449 $\mathrm{CPP}$ ), activités qui ont «notamment pour objet de développer les moyens d'expression, les connaissances et les aptitudes des détenus» (art. D $441 \mathrm{CPP}$ ). Ces droits culturels recouvrent notamment un droit à l'information, avec l'accès aux différents journaux et livres français et étrangers n'ayant pas fait l'objet d'une saisie dans les trois derniers mois (art. D $444 \mathrm{CPP}$ ), l'accès à la bibliothèque (art. D 441-2 CPP), ainsi que l'utilisation des postes de radio et de télévision loués par le biais de l'association socioculturelle (art. D $444 \mathrm{CPP}$ ). Le droit à l'enseignement et à la formation est également prévu, y compris par correspondance (art. D 450 sq. CPP), ainsi qu'un droit à l'expression écrite: des bulletins ou journaux rédigés par les détenus pouvant être diffusés à l'intérieur et à l'extérieur des établissements pénitentiaires avec l'accord et sous le contrôle de l'Administration (art. D 444-1 CPP). Le Code de procédure pénale prévoit également le droit à une pratique religieuse conforme aux convictions du détenu, grâce aux services d'un ministre du culte agréé (art. D 432 sq. CPP). Enfin, le règlement accorde aux détenus le droit, lorsqu'ils se trouvent dans leur cellule, de se livrer aux activités de leur choix « qui ne préjudicient pas à l'ordre et à la sécurité », ainsi que d'aménager leur cellule «de façon personnelle » (art. D 449 CPP).

Les droits des détenus sont donc aujourd'hui une réalité mais il reste à savoir si l'on peut dépasser le stade de liste de droits purement déclaratoires en les transformant en de véritables droits subjectifs. Seules des voies de droit et de recours adéquates, qui impliquent un renforcement de la légalité en prison, permettent une telle transformation.

\section{B. La progression de la légalité en prison}

La discussion ne porte plus tellement aujourd'hui sur l'existence de diverses prérogatives qui permettent au détenu, par des moyens divers, d'obtenir certains avantages, mais sur le tracé de la frontière entre ce qui constitue de véritables droits protégés et ce que l'on pourrait qualifier de simples «facultés » ou " prérogatives » offertes au détenu. Certes, l'existence d'un droit subjectif signifie bien un avantage, une prérogative à laquelle le bénéficiaire peut prétendre, mais qu'il peut également transmettre à d'autres, ou renoncer à exercer. Mais toutes les fois qu'existe un tel droit subjectif, son titulaire a droit à la protection qui s'exprime par la présence d'une action en justice venant le garantir. Il est aujourd'hui inexact de ne voir dans les établissements pénitentiaires que des lieux clos, en dehors du droit et soumis au pouvoir illimité des personnels de l'établissement (et notamment à la puissance tutélaire du chef de l'établissement).

On sait que la Cour européenne des droits de l'homme a synthétisé, par une formule devenue aujourd'hui célèbre, la position des détenus au regard de la Convention européenne des droits de l'homme: "La justice ne saurait s'arrêter à la porte des prisons ${ }^{27}$. Le détenu doit donc avoir la possibilité de défendre de manière directe tous ses droits contre les actes de l'Administration pénitentiaire devant des tribunaux indépendants. Se contenter de déclarer l'existence de droits risque fort d'être sans véritable intérêt pratique pour la situation réelle du prisonnier. L'application effective des droits des détenus suppose, en effet, un droit de plainte et la possibilité de former des recours administratifs ou judiciaires contre les décisions qui leur paraissent abusives ou mal fondées en $\operatorname{droit}^{28}$.

La situation du détenu en France présente une difficulté spécifique, qui s'explique en raison de la dualité de notre système juridique et de nos voies de recours contentieuses. La personne incarcérée se situe en effet dans

27. CEDH, 28 juin 1984, Campbell et Fell c. Royaume-Uni, série A, nº 80, p. 68, $\$ 69$. Afin de rendre effectif l'accès des personnes privées de liberté aux instances du Conseil de l'Europe, la Commission a estimé dès 1960 que la reconnaissance par un État de la compétence de la Commission pour connaître des requêtes introduites en application de l'article 25 de la Convention implique que les requérants ont le droit de correspondre librement avec elle, Requête $\mathrm{n}^{\circ} 538$ / 59, X c. RFA, inédit.

28. M. Barbarin, «Introduction au débat sur les régimes pénitentiaires et le traitement des détenus - la vie quotidienne en prison », in Actes des rencontres européennes pour les chefs des administrations pénitentiaires, Messine-Rome, 6-12 novembre 1989, p. 187. 
une position juridique précise, celle de l'utilisateur (certes contraint et forcé!) d'un service public national, le service public pénitentiaire. Les recours relatifs au fonctionnement de ce service public administratif relèvent classiquement de la compétence du juge administratif. Mais d'un autre côté, toutes les décisions qui concernent l'application et la durée de la peine ou de la mesure de détention préventive et qui sont prises par des magistrats de l'ordre judiciaire relèvent de la compétence du juge judiciaire. Cette dualité de contentieux est issue d'une décision du Tribunal des conflits de 1960 selon laquelle le juge administratif est compétent pour connaître des litiges relatifs à l'exécution du service public pénitentiaire par le personnel administratif et notamment par les directeurs des établissements ${ }^{29}$.

Or, jusqu'à une date récente, le juge administratif a été fort réticent à connaître des requêtes émanant de personnes privées de leur liberté. Les recours portés en matière pénitentiaire relevaient, pour l'essentiel, de la catégorie des «mesures d'ordre intérieur», pour lesquelles le juge administratif ne s'estimait pas compétent. Ainsi, « destinées à assurer la discipline dans les établissements pénitentiaires ${ }^{30}$, les sanctions disciplinaires relevaient-elles de cette catégorie, n'affectant pas le statut même des détenus et ne faisant donc pas grief. Il faudra attendre 1995 et l'affaire Marie pour que le Conseil d'État juge qu' " eu égard à la nature et à la gravité de cette mesure, la punition de cellule constitue une décision faisant grief susceptible d'être déférée au juge de l'excès de pouvoir ${ }^{31}$. Depuis cette date, le juge administratif contrôle l'exercice de nombreux droits en détention. C'est par exemple le cas d'une note de service refusant l'achat d'œufs en cantine, qui peut désormais être considérée comme un acte administratif faisant grief ${ }^{32}$... De même, les chefs d'établissements n'ont plus la possibilité de prononcer librement des mesures de sanction à l'égard de titulaires de permis de visites ${ }^{33}$. Mais c'est plus particulièrement le domaine des sanctions disciplinaires qui a été modifié par la possibilité de contester la sanction infligée par le chef d'établissement devant le juge administratif. Ainsi celui-ci vérifie désormais si l'administration apporte bien la preuve que la convocation par écrit du détenu pour le prononcé d'une sanction disciplinaire a été régulièrement effectuée au plus tard trois heures avant la réunion de la commission de discipline ${ }^{34}$, ou encore si apparaissent bien clairement quels étaient les agissements exacts que l'Administration a voulu sanctionner lors du prononcé d'une sanction disciplinaire ${ }^{35}$. En dehors du domaine strict des sanctions disciplinaires, le juge administratif a reconnu la recevabilité d'un recours contre un refus de décision de transfèrement dans une autre prison (application de la Convention européenne sur le transferrement du 21 mars 1983, du Conseil de l'Europe, entrée en vigueur le $1^{\text {er }}$ juillet $1985)^{36}$. Le juge administratif accepte même désormais de connaître des recours dirigés contre des mesures d'isolement prises à l'encontre des détenus (sur le fondement de l'article D 283-1 CPP) : en vertu d'une jurisprudence constante, le juge considérait que cette mesure n'aggravait pas les conditions de détention et ne pouvait donc faire l'objet d'un recours pour excès de pouvoir ${ }^{37}$. Cette analyse de l'isolement relevait d'une méconnaissance totale de la vie pénitentiaire, tant les conséquences désocialisantes et psychiquement déstructurantes d'une décision de mise à l'isolement ont été maintes fois exposées et dénoncées. Dans un arrêt du 20 juillet 2003, le Conseil d'État a enfin reconnu recevable une requête portée contre une mesure d'isolement, en considérant que le placement à l'isolement d'un détenu contre son gré constitue, eu égard à l'importance de ses effets sur les conditions de détention, une décision susceptible de faire l'objet d'un recours pour excès de pouvoir ${ }^{38}$.

Toutefois, la jurisprudence Marie et ses suites n'ont pas totalement fait disparaître la catégorie des mesures d'ordre intérieur et de nombreux pans de la vie pénitentiaire restent encore hors du contrôle du juge administratif. Sont ainsi toujours considérées comme des mesures d'ordre intérieur les décisions de transfert et d'affectation $^{39}$, le fichage d'un détenu comme détenu dangereux ${ }^{40}$, l'interdiction ponctuelle faite à un détenu de transmettre un courrier à un autre détenu ${ }^{41}$, le refus d'un chef d'établissement de reconfigurer les parloirs de telle manière que les condamnés puissent "entretenir des rapports conjugaux normaux avec leurs épouses ${ }^{42}$, la décision de faire quitter à un détenu la cellule qu'il occupait dans le quartier des travailleurs ${ }^{43}$, le placement d'un détenu

29. TC, 22 février 1960, Dame Fargeau D'Epied, Lebon, p. 853.

30. CE, 4 mai 1979, Comité d'action des prisonniers et autres, JCP, 1979, édition générale, II, 19242, concl. Franc., Dalloz, 1979, Information rapide, p. 390, obs. Delvolvé, Dalloz, 1980, p. 433, note M. Drapier.

31. CE, Ass., 17 février 1995, Marie; concl. P. Frydman, Revue française de droit administratif, $\mathrm{n}^{\circ}$ 11, 1995, p. 353 sq.

32. CAA Lyon, Hénaf, 13 décembre 2000, note É. Péchillon, «Actualité du droit de l'exécution des peines», Dalloz, 2002, p. 112.

33. CAA Douai, Aït-Taleb, 3 mai 2001, note É. Péchillon, ibid., p. 112-113.

34. TA Rouen, Aït-Taleb, 23 juin 1999.

35. TA Strasbourg, Lajoye, 20 janvier 1998.

36. TA Poitiers, $1^{\text {er }}$ juillet 1999, Morales c. garde des Sceaux, JCP, jurisprudence, II, 10 205, 1999, p. 2090 sq.

37. CE, 28 février 1996, Fauqueux. Sur l'isolement carcéral, voir notamment M. Herzog-Evans, «Quelques réformes récentes en droit pénitentiaire », Les Petites Affiches, 27 mai 1999, p. 16 sq.

38. CE, 30 juillet 2003, Garde des Sceaux, ministre de la Justice c. M. Saïd Remli.

39. CE, 8 décembre 1967, Kayanakis; confirmé plus récemment par TA Strasbourg, 20 février 1998, Rizzutti, TA Versailles, 15 décembre 2000, Remli.

40. CE, 12 novembre 1986, Winterstein.

41. CE, 8 décembre 2000, Frérot, Droit administratif, février 2001, p. 19-20.

42. TA Clermont-Ferrand, 15 décembre 2000, Remli, note M. Herzog-Evans, Dalloz, 2002, p. 114-115.

43. TA Nantes, 6 juillet 2000, Lahmar, ibid., p. 112. 
dans un quartier de plus haute sécurité ${ }^{44}$, ou la mise en cellule disciplinaire à titre préventif ${ }^{45}$. Le détenu usager du service public pénitentiaire n'apparaît donc toujours pas en mesure de faire protéger l'ensemble de ses droits par des voies de recours ouvertes et accessibles.

Les recours que le détenu condamné pouvait former contre les décisions du juge d'application des peines ${ }^{46}$ présentaient, jusqu'à une date récente, un parallélisme assez troublant avec les mesures d'ordre intérieur. Les décisions du juge d'application des peines étaient en effet prises après avis de la Commission d'application des peines ${ }^{47}$, selon une procédure non contradictoire, et n'étaient susceptibles d'aucun recours. Il faut attendre la loi du 31 décembre 1997 pour constater un premier assouplissement de cette fermeture du contentieux : les condamnés mis sous surveillance électronique peuvent faire appel, devant la Chambre des appels correctionnels, de la décision par laquelle le juge d'application des peines met fin à cette modalité d'exécution de la peine privative de liberté. Mais c'est surtout la loi du 15 juin $2000^{48}$ qui va instaurer un véritable droit de recours au profit du détenu, en exigeant que les décisions du juge d'application des peines soient motivées et en instaurant un débat contradictoire. Désormais, selon l'article 722 CPP, les mesures de placement à l'extérieur, de semi-liberté, de fractionnement et suspension des peines, de placement sous surveillance électronique et de libération conditionnelle sont accordées, ajournées, refusées, retirées ou révoquées par décision motivée du juge d'application des peines saisi d'office, sur la demande du condamné ou sur réquisition du procureur de la République. Cette décision est rendue, après avis du représentant de l'Administration pénitentiaire, à l'issue d'un débat contradictoire tenu en Chambre du conseil, au cours duquel le juge d'application des peines entend les réquisitions du ministère public et les observations du condamné ainsi que, le cas échéant, celles de son avocat. Mais surtout, cette décision peut être attaquée en appel par le condamné, le procureur de la République et par le procureur général, dans un délai de dix jours à compter de sa notification. L'appel est porté devant la Chambre des appels correctionnels ${ }^{49}$.

C'est également le domaine de la libération conditionnelle qui a connu des avancées récentes remarqua- bles. Introduite dans notre droit par la loi du 4 août 1885, cette mesure peut être décrite comme la mise en liberté anticipée d'un condamné, affectée d'une condition de bonne conduite jusqu'à l'expiration du délai convenu. La loi du 29 décembre 1972, modifiant l'article 730 CPP, avait prévu une dualité de compétence pour l'octroi de la libération conditionnelle : le juge d'application des peines pouvait accorder la libération conditionnelle aux condamnés subissant une peine privative de liberté dont la durée n'excédait pas cinq ans et le garde des Sceaux pouvait libérer les détenus condamnés à une peine supérieure à cinq ans. Ce dispositif avait eu l'occasion d'être critiqué à de nombreuses reprises, les commentateurs soulignant tout à la fois la lenteur de la décision, l'absence de procédure contradictoire de motivation des arrêtés et l'absence de voies de recours légales ${ }^{50}$... Il semblait, en outre, tout à fait critiquable de voir qu'un ministre pouvait ainsi intervenir dans l'exécution d'une peine privative de liberté prononcée par l'autorité judiciaire. Les différentes études menées au sujet de la libération conditionnelle montraient une situation préoccupante, les taux d'octroi de cette mesure diminuant régulièrement au cours des années. Ainsi, en dix ans, le nombre de libérations conditionnelles des condamnés relevant des juges d'application des peines était-il passé de 8167 en 1988 à 5098 en 1998. De même, le nombre de libérations conditionnelles des condamnés relevant de la compétence du garde des Sceaux était passé de 709 à 224 entre 1988 et $1998^{51}$. Cette tendance à la raréfaction des mesures de libération conditionnelle constituait une difficulté supplémentaire pour les personnels de surveillance de l'Administration pénitentiaire, devant gérer des détenus qui ne pouvaient compter sur aucun aménagement de peine et qui n'avaient aucune perspective, même lointaine, de libération. Avec la loi du 15 juin 2000, les conditions d'octroi de la libération conditionnelle sont devenues plus souples et le Code de procédure pénale n'exige plus que des «efforts sérieux de réadaptation sociale» pour que les détenus puissent bénéficier de la mesure $^{52}$. Mais surtout, avec la loi nouvelle, le ministre perd tout pouvoir en matière de libération conditionnelle et le juge d'application des peines voit son terrain d'intervention s'accroître. Désormais, ce magistrat est

44. CE, 27 janvier 1984, Caillol.

45. CE, 12 mars 2003, Garde des Sceaux, ministre de la Justice c. Frérot, commentaire de J.-P. Céré, Revue française de droit administratif, 2003 , p. 1012 sq.

46. Ce magistrat, présent auprès de chaque Tribunal de grande instance, est chargé de déterminer les principales modalités du traitement pénitentiaire. Selon l'article $722 \mathrm{CPP}$, il décide de mesures telles que la semi-liberté, le placement à l'extérieur, ou la libération conditionnelle des condamnés présents dans les établissements pénitentiaires du ressort de son tribunal.

47. Présidée par le juge d'application des peines, le procureur de la République et le chef de l'établissement pénitentiaire en sont membres de droit (art. $722 \mathrm{CPP}$ ).

48. Loi n ${ }^{\circ}$ 2000-516 du 15 juin 2000, JORF, 16 juin 2000 (en vigueur le $1^{\mathrm{er}}$ janvier 2001). Voir notamment M.-E. Cartier, «La juridiciarisation de l'exécution des peines ", Revue de science criminelle et de droit pénal comparé, 2001, p. 89 sq.

49. F. Le Gunehec, «Les deux étapes de la juridictionnalisation de l'application des peines », JCP, 24 janvier 2001, p. 173 sq.

50. Voir notamment, M.-E. Cartier, «Les propositions de la commission d'étude pour la prévention de la récidive », in Prison, sortir avant terme, J. Pradel (éd.), Paris, Cujas, 1996.

51. Voir Prisons: une humiliation pour la République. Depuis la réforme, le nombre de condamnés à plus de dix ans de prison, libérés en conditionnelle a un peu augmenté : 101 en 1999, 131 en 2000 et 167 en 2001. Les JAP ont quant à eux libéré 5361 détenus en 2000 et 5680 en 2001. Voir J. Coignard, «La conditionnelle, généralement sans récidive ", Libération, 9 octobre 2002.

52. À savoir notamment «lorsqu'ils justifient soit de l'exercice d'une activité professionnelle, soit de l'assiduité à un enseignement ou à une formation professionnelle ou encore d'un stage ou d'un emploi temporaire en vue de leur insertion sociale, soit de leur participation essentielle à la vie de famille, soit de la nécessité de subir un traitement, soit de leurs efforts en vue d'indemniser leurs victimes» (art. 729 CPP). 
compétent d'une part lorsque la ou les peines prononcées sont inférieures ou égales à dix ans et, d'autre part, lorsque quelle que soit la peine prononcée, il reste à subir une durée inférieure à trois ans. Mais la nouveauté la plus importante réside dans la création d'une nouvelle juridiction, établie auprès de chaque Cour d'appel et appelée Juridiction régionale de la libération conditionnelle. Elle a vocation à connaître tous les cas qui ne concernent pas la compétence du juge d'application des peines (à savoir les mesures relatives aux condamnés à une peine supérieure à dix ans et ceux pour lesquels la durée de détention restant à subir est supérieure à trois ans). Un appel peut être porté devant la Juridiction nationale de la libération conditionnelle. La libération de M. Patrick Henry par la Juridiction régionale de Basse-Normandie en mai 2001, alors que des demandes de mise en liberté avaient été déposées et rejetées à sept reprises, montre bien le changement radical initié par la loi du 15 juin 2000

Marqué par des avancées continuelles des droits reconnus aux personnes incarcérées et une multiplication des voies de recours, le droit pénitentiaire offre l'image d'une modernisation qui tend à faire du détenu un sujet de droit véritable. Toutefois, s'arrêter à des constatations n'aboutirait qu'à percevoir une partie de la réalité, car la situation du détenu ne doit pas être séparée des réalités carcérales qui aboutissent, dans les faits, à la limitation de l'exercice des droits en détention.

\section{Droits des détenus et réalités pénitentiaires}

La prison et le prisonnier sont bien appréhendés par le droit. Toutefois, cette branche juridique particulière que l'on a pris l'habitude d'appeler le droit pénitentiaire offre de nombreuses particularités et spécificités (A), qui sont encore renforcées par les conditions matérielles de l'exercice des droits (B).

\section{A. Le maintien des spécificités du droit pénitentiaire}

Si la prison doit officiellement transformer l'individu en lui apportant, lors de sa détention, les éléments qui prépareront au mieux sa réinsertion, il ne faut jamais perdre de vue l'une des autres fonctions de la peine privative de liberté. Cette mission, au cœur même de l'institution carcérale, est la recherche de la sécurité: "parce que l'administration pénitentiaire a pour première mission l'exécution des décisions d'emprisonnement prises par les juridictions, la sécurité de ses établissements ne saurait souffrir aucune défaillance... ${ }^{53}$. L'emprisonnement carcéral reste un moyen de retrancher de la société, pendant un temps, des individus qui ont causé un trouble majeur à l'ordre social. Les établissements sont là pour emprisonner des délinquants, plus ou moins dangereux, mais qui ont comme trait commun la nécessité d'être retirés pour un temps plus ou moins long du corps social. Pour se convaincre du maintien de l'objectif de sécurité en prison, la lecture du Programme pénitentiaire issu de la loi d'orientation et de programmation pour la justice (2003-2007), rendu public en novembre 2002, montre bien ce souhait sécuritaire: alors que les évolutions du statut du détenu ne sont pas évoquées, le chapitre consacré à la "prison du XXI ${ }^{\mathrm{e}}$ siècle » commence par des développements très détaillés sur «la prison plus sûre ${ }^{54}$... Comme l'indique É. Péchillon, «l'institution carcérale a développé une centralisation et une hiérarchisation absolue de ses missions au profit de la sécurité. Le droit a fini par reproduire cet ordonnancement en "sacralisant" la mission de sécurité, jusqu'à en figer la signification juridique ${ }^{55}$. L'analyse des droits du détenu ne doit jamais être dissociée de l'exigence de sécurité au sein des établissements pénitentiaires. Pour certains, l'exercice des droits du détenu est toujours subordonné «à ce qui est objectivement, voire subjectivement, compatible avec l'ordre, la discipline et la sécurité, les « droits » tendant alors à devenir des faveurs, des privilèges accordés ou retirés selon ces impératifs, voire à être annihilés ${ }^{56}$. Le maintien de l'ordre et de la discipline exigent des rapports hiérarchiques très clairs: le détenu doit obéissance aux fonctionnaires ou agents ayant autorité dans l'établissement pénitentiaire, «en tout ce qu'ils prescrivent pour l'exécution des règlements » (art. D 243 $\mathrm{CPP}$ ). Il se trouve donc dans une position de sujétion par rapport à l'Administration pénitentiaire et à ses différents personnels. L'ordre et la discipline «doivent être maintenus avec fermeté, mais sans apporter plus de contraintes qu'il n'est nécessaire pour le maintien de la sécurité et d'une bonne organisation de la vie en collectivité » (art. D 242 CPP). Voilà toute l'ambiguïté en la matière de la mission de l'Administration pénitentiaire...

Afin de maintenir le bon ordre et la sécurité des établissements, on ne s'étonnera pas que le Code de procédure pénale autorise le personnel pénitentiaire à utiliser la contrainte, comme par exemple le port de menottes ou d'entraves «par mesure de précaution contre les évasions ", pendant un transfèrement ou une extraction (art. D 283-4 CPP). Ce même personnel est également habilité à utiliser la force, « en cas de légitime défense, de tentative d'évasion ou de résistance par la violence ou par inertie physique aux ordres donnés » (art. D 283-5 CPP).

53. V. Pécresse, Avis présenté au nom de la Commission des lois constitutionnelles, de la législation et de l'administration générale de la République sur le projet de loi de finances pour 2003, t. VI, «Justice, administration pénitentiaire et protection judiciaire de la jeunesse ", n 291, 10 octobre 2002.

54. Source: http://www.justice.gouv.fr/presse/conf211102iii.htm. Voir également J.-M. Chauvet, Rapport sur la sécurité des établissements pénitentiaires et des personnels, rapport remis à Madame le garde des Sceaux, 19 octobre 2001.

55. É. Péchillon, Sécurité et Droit du service public pénitentiaire, Paris, LGDJ (Bibliothèque de droit public; t. 204$), 1998$.

56. J. Giudicelli-Delage et M. Massé, «Rapport introductif», in La Condition juridique du détenu, J. Pradel (dir.), Paris, Cujas (Travaux de l’Institut de sciences criminelles de Poitiers; vol. XIII), 1993, p. 19. 
L'utilisation de l'isolement constitue également un instrument au service de la sécurité des établissements ${ }^{57}$.

Au-delà de la recherche légitime de la sécurité des établissements, des personnes qui y vivent et qui y travaillent et de la société toute entière, la mise en avant de la sécurité dans les établissements constitue surtout un instrument aisé de limitation de l'exercice des droits conférés aux détenus. La recherche de la sécurité explique de nombreuses limitations implicites ou explicites à l'exercice de certains droits, modulations rendues d'autant plus aisées à opérer que le droit pénitentiaire utilise fréquemment des formules larges, sujettes à interprétation et qui laissent souvent un large pouvoir discrétionnaire aux personnels de l'établissement pénitentiaire pour leur application... Les règles pénitentiaires posent le plus souvent des principes, assortis de dérogations et de rédactions suffisamment souples, afin de ne pas entraver le bon fonctionnement des établissements ${ }^{58}$. Ainsi, pour les sénateurs, «dans le droit du "dehors", tout ce qui n'est pas explicitement défendu est autorisé; dans le droit du "dedans", tout ce qui n'est pas explicitement permis est interdit. Alors que le détenu est normalement privé de la seule "liberté d'aller et de venir", de nombreuses libertés sont supprimées en prison, ou inapplicables, en dehors même de celles qui deviennent inapplicables en raison de la surpopulation pénale ${ }^{59}$.

Il en résulte des régimes de détention et des droits variables selon les établissements. Ainsi les députés relèvent-ils en 2000 que «les visites des établissements pénitentiaires ont fait apparaître que les règles différaient totalement dans des domaines qui touchent de près la vie quotidienne des détenus. Il en est ainsi des durées de parloir qui peuvent varier de une heure à une durée illimitée ; de la pratique des fouilles qui se font avant et après chaque parloir, certains établissements pratiquant la fouille par palpation avant le parloir et d'autres la fouille à corps ; de l'accès au téléphone qui peut se faire sans limitation de durée par le biais de carte téléphonique et sans surveillance ou qui peut au contraire être très réglementé avec des conversations enregistrées ${ }^{60}$; de l'ouverture du courrier qui peut présenter un caractère systématique ou être [effectuée] simplement par sondage. Dans tous ces domaines, toutes les pratiques ont été rencontrées $"{ }^{61}$. L'exemple de la censure du courrier constitue un bon exemple de restriction dans l'usage d'un droit. Pour des raisons de sécurité bien compréhensibles, l'article D 416 CPP prévoit que «les lettres de tous les détenus, tant à l'arrivée qu'au départ, peuvent être lues aux fins de contrôle». Or, cette simple « possibilité » laissée à l'administration constitue souvent la règle de fonctionnement des établissements pénitentiaires. Certes, des exceptions sont prévues pour permettre le secret de la correspondance avec certaines autorités ${ }^{62}$, mais dès qu'un détenu voudra s'adresser à une telle autorité les surveillants lui demanderont sans nul doute pourquoi il souhaite le secret de sa correspondance. De même, le rapport sénatorial de 2000 sur les prisons indique que lors de la sortie du livre du docteur Véronique Vasseur ${ }^{63}$, on a pu constater la censure de certaines coupures de presse relatant la situation des établissements pénitentiaires et adressées en pièces jointes dans des correspondances aux détenus. Comment justifier ces mesures, alors que tout détenu a le droit, par ailleurs, de s'abonner à des journaux et magazines? Les articles D 431 et D 444 CPP prévoient en effet théoriquement la possibilité pour les détenus d'acheter toutes les publications légalement distribuées à l'extérieur.

Sans même parler des exigences de sécurité, les droits des personnes incarcérées sont presque toujours limités dans leurs fins, car conditionnés par l'exigence d'un traitement. Ils n'existent que parce que l'on estime que la société, ayant privé le détenu d'une partie de ses droits et l'ayant placé dans une situation de dépendance, a également le devoir de le protéger et de lui assurer certaines facilités, concernant la formation, l'emploi, les soins, etc. L'étendue de ces droits secondaires ou conditionnés est totalement liée à la qualité du traitement. Toutefois, la pratique montre que les restrictions à l'exercice des droits sont motivées tantôt par le souci, plus ou moins conscient, de conserver un certain caractère afflictif et pénible à la peine, tantôt par les nécessités pratiques de la vie dans une institution fermée, mais assez rarement dans le seul but du traitement. On peut ainsi se demander légitimement pourquoi le travail pénal est, dans la réalité carcérale, souvent dévalorisant et très mal rémunéré, pourquoi des visites quotidiennes ne sontelles pas possibles, pourquoi restreint-on les loisirs, l'autorisation de téléphoner ${ }^{64}$ et d'envoyer du courrier,

57. Art. D 283-1 CPP : «Tout détenu se trouvant dans un établissement ou quartier en commun peut soit sur sa demande, soit par mesure de précaution ou de sécurité, être placé à l'isolement $[\ldots .$. .»

58. Cette constatation se trouve aggravée par une particularité juridique du droit pénitentiaire : le cadre législatif de la détention, posé par le code de procédure pénale, est particulièrement bref. Le droit pénitentiaire est en effet avant tout de nature réglementaire (art. D 50 à D 519 CPP). Les circulaires, mais également les règlements de chaque établissement, jouent un rôle primordial en ce qui concerne la source des droits de personnes incarcérées.

59. Voir Prisons: une humiliation pour la République.

60. Sur l'utilisation du téléphone par les détenus en France, le Comité de prévention de la torture affirmait en 1996 qu' «il va de soi que, dans l'intérêt des instructions judiciaires, il peut être nécessaire d'interdire à un prévenu des contacts avec le monde extérieur pendant un certain temps. De plus, dans certains cas, le magistrat instructeur pourrait soumettre les contacts téléphoniques à un contrôle approprié. Toutefois, interdire à tout prévenu pendant toute la durée de détention de tels contacts est, de l'avis du Comité de prévention de la torture, injustifié. L'on ne peut du reste que noter qu'une telle approche s'éloigne de celle suivie dans d'autres pays européens ». Comité européen pour la prévention de la torture et des peines ou traitements inhumains ou dégradants, Rapport au Gouvernement de la République française relatif à la visite effectuée par le CPT en France du 6 au 18 octobre 1996 , p. 60.

61. Rapport de M. J. Floch (cf. note 4).

62. Comme la correspondance adressée aux avocats (art. D 69 CPP) ; la correspondance adressée aux autorités administratives et judiciaires (art. D 262 CPP) et aux aumôniers (art. D 438).

63. V. Vasseur, Médecin-chef à la prison de la Santé (Paris, Le Cherche Midi, 200o).

64. L'avant-projet de loi pénitentiaire, préparé par les services de $\mathrm{M}^{\mathrm{me}}$ Lebranchu en 2001, prévoyait sur cette question que l'usage du téléphone, jusqu'ici réservé dans la pratique aux longues peines, aurait été accessible aux condamnés et aux prévenus, avec toutefois des conversations «systématiquement 
pourquoi refuse-t-on au détenu la possibilité de diriger une entreprise? Les droits reconnus au détenu restent limités par des contraintes carcérales multiples, pas toujours évidentes à appréhender de l'extérieur. Ainsi, la partie réglementaire du Code de procédure pénale permet au directeur d'établissement de refuser un permis de visite ou d'imposer le dispositif de parloir avec séparation; un surveillant peut mettre fin à un entretien au parloir $^{65}$; un directeur d'établissement peut décider de « déclasser » un détenu qui travaille suite à une infraction commise à l'occasion du travail (art. D 251-1 CPP).

Les droits reconnus aux détenus sont pour l'essentiel finalisés, admis en fonction d'un usage déterminé. Ceux qui déclarent que les détenus n'ont pas de droits font une analyse inexacte de la situation, en confondant la reconnaissance de droits et leur maîtrise et garantie. Le statut du détenu ne le prive pas de droits, mais ne lui reconnaît que ceux nécessaires à la limitation des effets pervers de l'incarcération ou à sa réinsertion future. La personne incarcérée n'est étrangère qu'à l'idée de droits égoïstes. Si l'on veut tenter de transposer la situation du détenu à celle d'une autre catégorie, la comparaison la plus juste semble être d'assimiler le prisonnier contemporain à un mineur, voire à un majeur sous tutelle. Dans les deux cas, le sujet de droit possède une autonomie juridique réduite et est soumis à un élément extérieur qui peut le contraindre et décider en ses nom et place: le directeur de l'établissement pour l'un, le titulaire de l'autorité parentale ou le tuteur pour l'autre. Il est indéniable que la prison constitue un instrument d'infantilisation du prisonnier, totalement pris en charge, soigné, nourri, traité, formé, employé... Le prisonnier est soumis à un traitement, mais ne possède aucun droit de regard sur ce traitement, tout comme l'enfant ne possède que peu de droits sur son éducation. Les normes pénitentiaires décident ce qui est «bon" pour le détenu: qui seront les destinataires souhaitables de sa correspondance, jusqu'à quel point il peut disposer de son corps, quelle est la forme de travail la plus utile à sa réinsertion, quelle est la part de sa rémunération qui doit être consacrée à la constitution d'un pécule, etc. La notion de «mesures d'ordre intérieur» ne fait d'ailleurs que traduire en termes juridiques cette philosophie rééducatrice de la prison. Si le directeur de l'établissement censure le courrier à destination du détenu ou interdit certaines visites, la motivation est certainement d'éviter que ne parviennent à ce prisonnier des nouvelles de la société criminogène dans laquelle il baignait avant l'incarcération ou que de mauvaises fréquentations ne viennent la lui rappeler ${ }^{66} \ldots$ Si le détenu mineur ou anal- phabète est contraint de suivre des cours, c'est uniquement parce que ses compétences lui serviront à ne point tomber dans la récidive. De quel droit le détenu contesteraitil la prise de telles mesures?

Droits contraints par la recherche de la sécurité, droits orientés par l'exigence du traitement pénitentiaire, les droits du détenu sont également des droits qui ne peuvent être en croissance infinie. Le mouvement évoqué jusqu'à présent semble être celui d'une progression inéluctable des droits des personnes incarcérées, qui se caractériserait par une reconnaissance de droits multiples, assortie de voies de recours adéquates et effectives. Or, ce mouvement se heurte à ce que l'on pourrait appeler le principe de «proportionnalité » des droits de la personne incarcérée. C'est Jeremy Bentham qui a fait ressortir le premier la nécessaire adéquation entre les droits reconnus à la personne détenue (« la condition ordinaire d'un prisonnier », selon la terminologie retenue par Bentham) et ceux dont jouissent les individus libres de sa classe : "sauf les égards dûs à la vie, à la santé, et au bienêtre physique..., on ne doit pas rendre sa condition meilleure que celle des individus de cette même classe qui vivent dans un état d'innocence et de liberté $"{ }^{67}$. Il s'agit là, posé dès 1791, d'un élément fondamental de théorie en ce qui concerne les droits de la personne incarcérée : la peine de prison doit certes s'effectuer dans le plus grand respect de la personne détenue, mais cette «douceur» de la peine doit toujours être mise en perspective avec les droits de l'individu libre, sous peine d'ôter à la peine de prison son caractère utilitaire et donc de faire droit aux théories des abolitionnistes. Cette idée forte a été rappelée très récemment par un observateur avisé des prisons françaises, Robert Badinter, devant la Commission d'enquête de l'Assemblée nationale: «J'en arrive à ce qui domine, à mon sens, le problème. Une loi d'airain pèse sur la prison. Je l'ai appelée "loi d'airain", car je ne l'ai jamais vue démentie: vous ne pouvez pas, dans une société démocratique déterminée - je ne parle pas des prisons totalitaires, car l'idée même de respect de la dignité humaine n'existe pas - porter le niveau de la prison au-dessus du niveau de vie du travailleur le moins bien payé de cette société. Le corps social ne supporte pas que les détenus vivent mieux que la catégorie sociale la plus défavorisée de la société ${ }^{68}$.

Théorie et pratique se rejoignent donc pour limiter l'exercice des droits par la personne incarcérée. Mais il existe un autre élément de limitation, qui réside dans les conditions matérielles et concrètes de l'exercice des droits.

66. Ce que reconnaît également la Cour européenne des droits de l'homme : l'isolement relatif découlant de l'interdiction, pour des raisons de sécurité, d'avoir des contacts avec des détenus sous le régime normal, de recevoir des visites, hormis de sa famille, et de téléphoner n'était pas, en lui-même, constitutif d'une forme de traitement inhumain. En l'espèce, Le requérant avait été soumis à ce régime en raison de ses liens étroits avec le milieu mafieux. L'interdiction de participer aux activités récréatives se justifiait dans la mesure où celles-ci auraient pu lui permettre de reprendre contact avec ce milieu. CEDH, 8 juin 1999, A.M. c. Italie.

67. J. Bentham, Le Panoptique, Paris, Belfond, 1977, p. 20.

68. Rapport de M. J. Floch (cf. note 4). 


\section{B. L'exercice des droits du détenu face à la réalité carcérale}

Les droits des personnes incarcérées présentent de nombreuses particularités théoriques, mais leur exercice concret doit également attirer l'attention. Leur étude purement positiviste ne peut en aucun cas rendre compte de la réalité pénitentiaire. Ces droits doivent toujours être mis en situation et examinés dans leur application. À ce titre, il ne faut jamais perdre de vue la situation concrète des établissements pénitentiaires. La prison étant par essence un lieu clos, on sait que l'exercice des droits s'analyse souvent en un rapport de force entre détenus et personnel de surveillance. Mais ce sont encore davantage les conditions matérielles de détention qui posent problème et la situation des prisons françaises constitue un sujet excessivement préoccupant. Les regards récents portés sur la prison ont tous abouti à des exposés extrêmement négatifs et critiques. Véronique Vasseur, médecin-chef à la prison de la Santé, décrivait ainsi cet établissement: "Je découvre d'abord des murs écaillés, des chasses d'eau qui fuient, couvertes de mousse, des détritus par terre, des fientes de pigeon, des plumes, des milliers de pelures d'orange pendues aux barreaux pour masquer les effluves des W.-C. à l'intérieur des cellules [...]. Les cellules font dix mètres carrés et demi et accueillent trois ou quatre détenus. Les murs sont de couleur papier kraft avec une petite ampoule de 60 watts, à trois mètres du sol. Ils suintent de salpêtre. La fenêtre est minuscule et aucun air ne circule. Les carreaux cassés ne sont pas changés, le W.-C. collectif n'a même pas de paravent et on s'étonne qu'ils soient tous constipés. Essayez de déféquer devant trois inconnus! La vermine envahit les matelas " ${ }^{69}$. La visite effectuée par le Comité européen contre la torture à la maison d'arrêt de la Santé à Paris ne décrit malheureusement pas une autre situation: "Quant aux Divisions B, C et D, les cellules étaient dans un état de dégradation très avancé, comme les bâtiments mêmes dont le gros œuvre était attaqué. Leur équipement était à l'identique (lits vétustes, matelas / couvertures sales et usées). En particulier, le lavabo et les toilettes des cellules (camouflés derrière un rideau de fortune) étaient délabrés et insalubres - sans même évoquer l'odeur se dégageant des toilettes. En outre, les cellules étaient infestées par des poux et d'autres vermines; la présence de rongeurs n'était pas non plus exceptionnelle. C'était là une plaie contre laquelle l'établissement avait beaucoup de mal à lutter ; le problème des rongeurs, en effet, était aigu dans l'arrondissement où se situe la maison d'arrêt. Quant aux douches desservant ces divisions, la situation n'était guère meilleure, en dépit de certains travaux ponctuels. À leur état de dégradation et d'insalubrité, s'ajoutait celui de leur saleté. En résumé, les conditions matérielles de détention dans les divisions $\mathrm{B}, \mathrm{C}$ et $\mathrm{D}$ étaient misérables et comportaient des risques pour la santé des détenus. Dans l'ensemble de l'établissement, les dispositions prises pour permettre aux détenus d'assurer la propreté de leur cellule et leur hygiène corporelle n'étaient pas satisfaisantes. Les produits nécessaires à l'entretien des cellules n'étaient pas fournis. En outre, les produits d'hygiène corporelle de base n'étaient disponibles qu'en quantité insuffisante. Les détenus se voyaient contraints de cantiner pour ces objets, et ceux qui étaient sans ressources devaient compter sur les autres détenus. À cet égard, le directeur de l'établissement a indiqué vouloir faire figurer cette question parmi les priorités de fin d'année ${ }^{70}$. Les sénateurs n'ont pas vu autre chose en visitant la prison de la Santé: «certaines douches de la maison d'arrêt [...] sont sordides : le plafond est couvert de salpêtre, la peinture a disparu, il manque le carrelage ${ }^{71}$...

La qualité des établissements pénitentiaires constitue donc un problème particulièrement préoccupant: 109 établissements ont été construits avant 1920, dont 23, avant 1830, accueillent encore environ 2800 détenus. En outre, 45 établissements sont installés dans des anciens couvents ou des casernes désaffectées. Au surplus, l'occupation de ces établissements constitue un autre sujet d'inquiétude: au $1^{\text {er }}$ novembre 2002, 54438 personnes étaient détenues dans les établissements pénitentiaires pour une capacité d'accueil de 47933 places, soit un taux d'occupation de $114 \%{ }^{72}$. La surpopulation carcérale constitue donc une réalité, même si elle varie de manière considérable entre les établissements ${ }^{73}$.

Les conditions matérielles de détention influent directement sur les droits qui devraient être normalement reconnus aux personnes incarcérées. D’une manière générale, les bâtiments ne répondent plus aux exigences modernes de conditions de détention dignes et humaines. La surpopulation carcérale s'oppose tout d'abord aux dispositions du Code de procédure pénale qui prévoient l'isolement individuel de jour comme de nuit dans les maisons d'arrêt ${ }^{74}$. La même constatation s'impose pour les dispositions du Code de procédure

69. V. Vasseur, Médecin-chef à la prison de la Santé, p. 49

70. Extrait du Rapport au Gouvernement de la République française relatif à la visite effectuée par le CPT en France du 6 au 18 octobre 1996, \$\$99 à 107. Lors de la visite effectuée dans le même établissement en 2001, le Comité a cependant " constaté un certain nombre de progrès dans les conditions matérielles de détention ». Voir Rapport au Gouvernement de la République française relatif à la visite effectuée par le CPT en France du 14 au 26 mai 2000 , \$80.

71. Prisons: une humiliation pour la République.

72. Programme pénitentiaire issu de la loi d'orientation et de programmation pour la justice, 203-2007, source: http://www.justice.gouv.fr/presse/ conf211102iii.htm.

73. Elle touche surtout les maisons d'arrêt : le rapport entre le nombre de détenus et le nombre de places opérationnelles (densité de population carcérale) est, au total, de 98,4 détenus pour 100 places au $1^{\text {er }}$ janvier 2001, mais il atteint $105 \%$ en maisons et quartiers de maison d'arrêt. 35 maisons ou quartiers de maison d'arrêt ont une densité supérieure à $150 \%$ (contre 40 en 1999). Voir Rapport annuel d'activité de l'Administration pénitentiaire, 200o, Ministère de la justice, 2002; http://www.ladocumentationfrancaise.fr/brp/notices/024000162.shtml.

74. L'article D 83 CPP prévoit toutefois de manière prudente que ce régime de l'emprisonnement individuel de jour et de nuit est appliqué « dans toute la mesure où la distribution des lieux le permet »... 
pénale qui préconisent pour les locaux de détention le respect des « exigences de l'hygiène, compte tenu du climat, notamment en ce qui concerne le cubage d'air, l'éclairage, le chauffage et l'aération » (art. D 350 CPP) et qui sont directement contredites par l'occupation de cellules de petite taille par plusieurs détenus ${ }^{75}$. L'absence d'eau chaude en cellule peut-elle, au XXI' siècle, être considérée comme compatible avec des installations sanitaires «décentes» (art. D $351 \mathrm{CPP})$ ? Les sénateurs rapportent également la question de la propreté de la literie: les draps sont changés une fois par semaine à la maison d'arrêt de Saint-Malo, mais seulement une fois par mois à celle de Rochefort. De même, la maison d'arrêt de Melun change les couvertures une fois par mois à la demande, mais celles de la maison d'arrêt de Reims ne sont changées qu'une fois par an ${ }^{76}$. Dans de telles conditions, on voit mal comment le détenu pourrait respecter l'article D 249-3 qui voit dans le fait de «négliger de préserver ou d'entretenir la propreté de sa cellule ou des locaux communs » une faute disciplinaire de troisième catégorie ! On doit d'ailleurs remarquer que le droit positif prend lui-même en considération les contraintes liées aux établissements. Ainsi, si «l'établissement doit être doté d'équipements sportifs de plein air et couverts, réglementaires et polyvalents, permettant l'organisation de séances et de rencontres sportives » (art. D 459-2 CPP), cette même disposition prévoit préalablement que ceci s'applique «sous réserve des contraintes architecturales "... Seules des conditions de détention compatibles avec le respect de la dignité humaine pourront permettre l'exercice effectif des droits des détenus ${ }^{77}$. Toutefois, la construction d'établissements neufs ne résout pas systématiquement tous les problèmes. En particulier, la construction des six établissements prévus dans le programme 4000 s'est effectuée essentiellement hors des zones urbaines. Or, en 2000 un seul de ces sites était desservi par les transports en commun. Le droit au maintien des relations avec l'extérieur pour le détenu apparaît alors bien problématique lorsque les visiteurs, pour un parloir d'une heure et demie, doivent se libérer une journée entière et utiliser soit leur véhicule personnel soit payer un taxi. De même, certains établissements peinent à faire venir des enseignants extérieurs en raison des contraintes liées à l'éloignement ${ }^{78}$.
Les différences entre les conditions de détention rendent le principe d'égalité des détenus devant le service public pénitentiaire totalement inopérant. Chaque établissement a ses spécificités propres, qui ressortent de son règlement intérieur, mais également des pratiques, de la politique plus ou moins libérale du directeur de l'établissement, des caractéristiques de l'établissement et de la population carcérale, etc ${ }^{79}$. L'utilisation de téléphones portables, les relations sexuelles lors de visites au parloir, normalement interdites, sont ainsi parfois tolérées dans certains établissements, mais peuvent faire l'objet de sanctions disciplinaires dans d'autres. De telles applications divergentes du droit ne peuvent que renforcer l'impression d'arbitraire.

Ces multiples inégalités renforcent la nécessité de prévoir, au-delà des droits reconnus et des voies de recours adéquates, des contrôles effectifs sur ce qui se passe à l'intérieur des établissements. En théorie, les prisons sont soumises à des contrôles extrêmement variés : l'Inspection des services pénitentiaires et l'Inspection générale des services judiciaires interviennent chacune dans leur domaine de compétence. Plus généralement, l'article D 231 CPP prévoit que «les administrations ou corps intéressés par certaines parties du service des établissements pénitentiaires sont habilités à en vérifier l'organisation et le fonctionnement, dans la limite des attributions que leur confèrent les lois et règlements ». Ainsi, l'Inspection du travail, comme l'Inspection générale des affaires sociales ou l'Inspection générale de l'Éducation nationale peuvent intervenir en prison. En outre, il existe une commission de surveillance auprès de chaque établissement pénitentiaire, commission qui « est chargée de la surveillance intérieure de l'établissement pénitentiaire en ce qui concerne la salubrité, la sécurité, le régime alimentaire et l'organisation des soins, le travail, la discipline et l'observation des règlements, ainsi que l'enseignement et la réinsertion sociale des détenus » (art. D 184 CPP). Les magistrats sont également conviés à cette vaste mission de contrôle des établissements pénitentiaires : l'article 727 CPP prévoit que «le juge de l'application des peines, le juge d'instruction, le président de la Chambre d'accusation [...], le procureur de la République et le procureur général visitent les établissements pénitentiaires ». Il faut désormais ajouter à ce

75. La Commission d'enquête parlementaire rapporte le cas de la prison de Gradignan, où un député membre de la Commission a pu constater que trois détenus vivaient dans $6 \mathrm{~m}^{2} 21$ heures sur 24. Voir le rapport de M. J. Floch sur la situation dans les prisons françaises (cf. note 4).

76. Les sénateurs ajoutant d'ailleurs, avec un soupçon d'humour noir, que «les détenus "sans famille" doivent laver leur linge en cellule, ce qui nécessite de l'eau chaude, ou éventuellement sous la douche (exemple de Fleury-Mérogis), puis le faire sécher devant les barreaux, ce qui donne, vu des coursives, une vision parfois pittoresque et confère à certaines de nos maisons d'arrêt un caractère napolitain ». Voir Prisons: une humiliation pour la République.

77. Un mouvement général de construction et de rénovation d'établissements pénitentiaires a été lancé en 1988 (programme 4000 ) et réactivé par le Gouvernement Raffarin à partir de 2002. Ce programme prévoit la construction de 22 nouveaux établissements pour les détenus majeurs (dont deux dans les DOM-TOM) et de 8 établissements spécialisés pour les mineurs, en application des engagements pris dans le cadre de la loi d'orientation de la justice. Ceci débouchera sur l'ouverture de 13200 nouvelles places de prison, sera financé à hauteur de 1,4 milliard d'euros et devrait être livré au plus tard en 2007.

78. Voir, à ce titre, O. Clingman, L. Gratiot, J.-C. Hanoteau, Le Droit en prison, qui mentionne le cas de Clairvaux où n'est présent qu'un seul instituteur et de Château-Thierry où aucun enseignant n'a accepté d'intervenir (p. 273).

79. La Commission nationale consultative des droits de l'homme a, dans son avis en date du 17 juin 1999, demandé que «soit entreprise une harmonisation nationale des règlements intérieurs selon des critères généraux établis en fonction des types d'établissements, afin qu'il soit mis un terme à une situation dans laquelle la disparité entre les règlements intérieurs des différents établissements, édictés au gré de chaque direction, entraîne une inégalité dans l'application de la loi ». 
tableau très dense des autorités de contrôle les députés et sénateurs, qui « sont autorisés à visiter à tout moment les locaux de garde à vue, les centres de rétention, les zones d'attente et les établissements pénitentiaires » en vertu de la loi du 15 juin 2000 renforçant la protection de la présomption d'innocence et les droits des victimes (art. 129). Le nombre et la variété de ces contrôles ne peuvent laisser penser que les établissements pénitentiaires restent des structures fermées, hors du regard de la société. Or, l'un des points communs de ces différents contrôles et inspections est justement leur inefficacité, pour des raisons multiples, mais qui peuvent toutes laisser penser à un grand désintérêt de ces acteurs vis-à-vis de la situation des personnes incarcérées ${ }^{80}$. Le premier Président de la Cour de cassation, Guy Canivet, a d'ailleurs dressé dans son rapport un bilan sans concession : "Ces contrôles ou inspections, y compris ceux des magistrats, ne sont pas satisfaisants, ni en quantité, ni en qualité, ni en cohérence. Ils n'ont pas toujours permis la révélation de manquements graves à l'intérieur d'une prison [...]. Les contrôles et inspections ne sont pas, dans l'ensemble, exercés de manière satisfaisante ${ }^{81}$. Le seul contrôle efficace, car à la fois spécialisé et indépendant, semble être aujourd'hui celui du Comité européen pour la prévention de la torture et des peines ou traitements inhumains ou dégradants ${ }^{82}$, dont les visites dans les établissements pénitentiaires français en 1991, 1994, 1996 et 2000 ont permis de mettre à jour de nombreux dysfonctionne- ments ${ }^{83}$. Même si le suivi des recommandations formulées reste délicat à assurer, le Comité est néanmoins l'image de ce que les différentes structures de contrôle et d'inspection pourraient apporter si elles étaient davantage effectives...

La place de l'enfermement carcéral au cœur des sanctions pénales n'apparaît pas réellement contestée aujourd'hui et se trouve même renforcée à la lumière de certaines évolutions législatives récentes. L'absence de réflexion d'ensemble sur les droits des détenus apparaît d'autant plus choquante. L'annonce par M. Lionel Jospin, lors de l'inauguration de l'École nationale de l'Administration pénitentiaire à Agen en novembre 2000, de l'élaboration d'une grande loi pénitentiaire avait pu à ce titre susciter de vifs espoirs, chez les détenus comme chez les personnels. Même si l'on peut penser qu'il ne s'agissait que de pures déclarations d'intention, le projet prévoyait d'inscrire au sein du Code pénal que «le recours aux peines privatives de liberté n'est possible que s'il constitue l'unique moyen de parvenir aux objectifs » que sont la protection de la société, la punition, la réinsertion, et les intérêts des victimes ${ }^{84}$. L'avant-projet de loi prévoyait, en outre, d'insister sur l'objectif d'insertion des détenus ${ }^{85}$. Même si ce projet semble aujourd'hui largement enterré, il s'agissait pourtant de la seule voie praticable, si l'on veut éviter de voir l'emprisonnement redevenir une peine strictement éliminatrice, sans autre fonction que celle de retrancher les délinquants aux yeux de la société.

80. Les sénateurs évoquent à cet égard l'exemple presque caricatural de la prison de Nice, où malgré plusieurs visites de la Direction des services vétérinaires et de la Direction des affaires sanitaires, il a fallu attendre une intoxication alimentaire massive des détenus le 6 janvier 1998 pour que le Préfet des Alpes-Maritimes décide de la fermeture des cuisines le 14 janvier 1998. Voir Prisons: une humiliation pour la République.

81. G. Canivet, L'Amélioration du contrôle..., p. 85.

82. En vertu de l'article $1^{\text {er }}$ de la Convention pour la prévention de la torture et des peines ou traitements inhumains ou dégradants du 26 novembre 1987 «le Comité examine le traitement des personnes privées de liberté en vue de renforcer, le cas échéant, leur protection contre la torture et les peines ou traitements inhumains ou dégradants".

83. En particulier en 1996, suite à une visite au Centre des jeunes détenus de Fleury-Mérogis, le Comité avait eu vent d'une enquête administrative discrète concernant un viol collectif dénoncé par un travesti. Le CPT avait alors alerté la Chancellerie qui avait saisi le Parquet d’Évry. L'affaire s'est terminée par la condamnation de 3 surveillants de Fleury à 4 ans de prison, dont 3 ans fermes. Voir E. Inciyan, « Trois surveillants de la prison de Fleury-Mérogis condamnés pour agressions sexuelles sur des travestis », Le Monde, 3 février 1999.

84. D. Simonnot, «Prisons : la sécurité avant la charité», Libération, 19 octobre 2001.

85. A. Gérin, Avis présenté au nom de la Commission des lois constitutionnelles, de la législation et de l'administration générale de la République sur le projet de loi de finances pour 2002, t. VI, «Justice, services pénitentiaires et protection judiciaire de la jeunesse», 11 octobre 2001. 
